\title{
Axisymmetric crack problem of thick-walled cylinder with loadings on crack surfaces
}

\author{
Levent Aydin *, H. Secil Altundag Artem \\ Department of Mechanical Engineering, Izmir Institute of Technology, Gulbahce Koyu, 35430 Urla-Izmir, Turkey
}

Received 14 April 2006; received in revised form 13 July 2007; accepted 16 July 2007

Available online 2 August 2007

\begin{abstract}
This study is concerned with the fracture of an infinite thick-walled cylinder. The inner surface of the cylinder is stress free and the outer is rigidly fixed. The cylinder having a ring-shaped crack located at the symmetry plane is subjected to distributed compressive load on its surfaces. The Hankel and Fourier transform techniques are used for the solution of the field equations. By applying the boundary conditions, the singular integral equation in terms of crack surface displacement derivative is derived. By using an appropriate quadrature formula, the integral equation is reduced to a system of linear algebraic equations. Numerical results are obtained for the stress intensity factors at the edges of the crack, surfaces of which are subjected to uniform, linear and parabolic load distributions.
\end{abstract}

(C) 2007 Elsevier Ltd. All rights reserved.

Keywords: Ring-shaped crack; Stress intensity factor; Integral transform; Singular integral equation

\section{Introduction}

In fracture mechanics, pressure vessels, pipes and other cylindrical containers having crack have been studied by numerous authors because of their industrial significance. No matter how a crack is initiated or introduced in a structural member, accurate prediction of the fracture conditions such as stress intensity factors, is important to assess its remaining life. One of the most widely used method of solution of crack problems leading to calculation of the stress intensity factors in infinite domains is the integral transform. A thick-walled cylinder with an axisymmetric internal or edge crack has been studied by Erdol and Erdogan [1]. The problem is formulated in terms of a singular integral equation which has a simple Cauchy Kernel for the internal crack and a generalized Cauchy Kernel for the edge crack.

Sneddon and Welch [2] analyzed the distribution of stress in a long cylinder of elastic material when it is deformed by pressure to the inner surfaces of a penny-shaped crack located symmetrically at the center of the cylinder. In the paper by Gupta [3] the axisymmetric semi-infinite cylinder with fixed short end is considered.

\footnotetext{
* Corresponding author.

E-mail address: leventaydin@iyte.edu.tr (L. Aydin).
} 


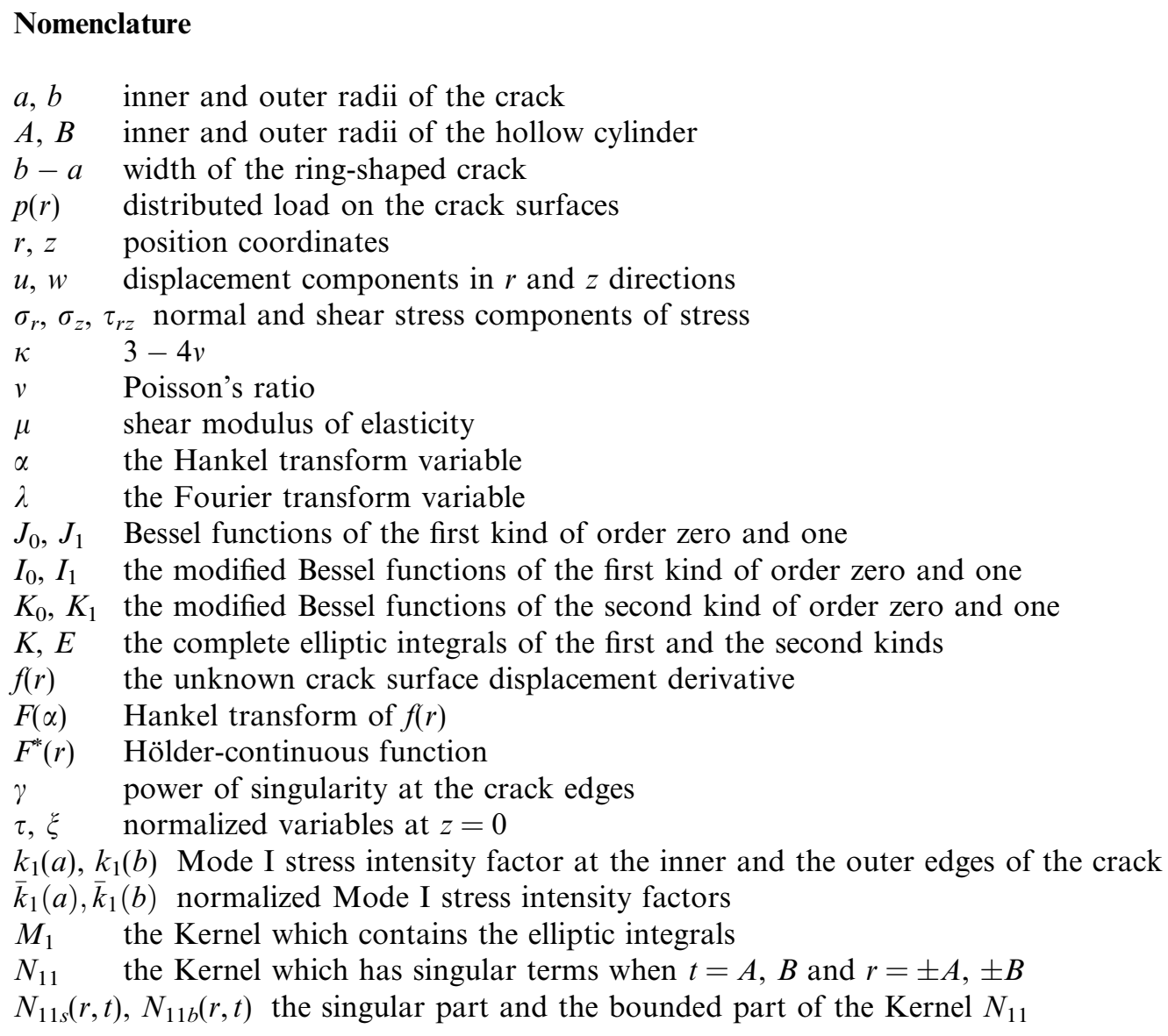

Integral transform method is used in order to formulate the problem and a singular integral equation is obtained. Delale and Erdogan [4] have considered a hollow cylinder problem. The cylinder contains an arbitrarily oriented radial crack and it is subjected to arbitrary normal tractions on the crack surfaces. In that study, the problem is formulated in terms of a singular integral equation using the basic dislocation solutions as the Green's functions. The elasticity problem for a long hollow cylinder which contains an axisymmetric circumferential crack has been studied by Nied and Erdogan [5]. The problem is formulated in terms of a system of singular integral equations with the Fourier coefficients of the derivative of the crack surface displacement density functions. The stress intensity factors (SIFs) and the crack opening displacement have been calculated. In the paper by Benthem and Minderhoud [6] the problem of a finite strip compressed between two rigid stamps is solved by the eigenfunction technique. Artem and Gecit [7] considered the fracture of an axisymmetric hollow cylindrical bar containing a ring-shaped crack at the symmetry plane and rigid inclusions. The cylinder is under the action of uniform loading. An integral transform technique has been used in order to formulate the problem in terms of the system of three singular integral equations. These integral equations are solved numerically and the stress intensity factors are calculated for crack and two rigid inclusions. Birinci [8] analyzed the elastostatic axisymmetric problem for a long thick-walled cylinder containing an axisymmetric circumferential internal or edge crack with cladding at the inner surface of the cylinder. An integral transform technique is used and the problem is formulated in terms of a singular integral equation. This integral equation is solved numerically by using quadrature formulas. The stress intensity factors are calculated and influence of the geometrical configuration and the cladding on the SIFs are discussed. A problem of ring-shaped crack contained in an infinitely thick-walled transversely isotropic cylinder is considered by Uyaner [9]. The cylinder is under the action of uniform loading. The stress function is expressed in terms 
of governing equations. Hankel and Fourier transforms are used and the problem is reduced to a singular integral equation. The singular integral equation is solved by the Gaussian quadrature and the stress intensity factors are calculated.

The present paper investigates the stress intensity factors at the edges of the crack for the long hollow cylinder containing a ring-shaped crack whose surfaces are subjected to distributed load. The hollow cylinder has rigid outer surface and stress free inner surface. The material of the cylinder is assumed to be homogeneous, linearly elastic and isotropic. General expressions for the problem is obtained by adding the solutions of two sub-problems: (1) an infinite medium having a ring-shaped crack and (2) an infinite medium without crack subjected to arbitrary axisymmetric loads, in order to have sufficient number of unknowns in the expressions. Displacement and stress expressions for the problems (1) and (2) are obtained by using the Hankel and the Fourier transform techniques, respectively. Applying the boundary conditions on the lateral surfaces of the cylinder and the crack surfaces, a singular integral equation in terms of crack surface displacement derivative is derived. The singular integral equation is reduced to a system of linear algebraic equations by using GaussLobatto quadrature formula. The improper integrals appearing in the coefficients are calculated numerically by using Gauss-Laguerre integration formula. Finally, normalized Mode I stress intensity factors at the edges of the crack are calculated. The main objective of this study is to have a good acquaintance with the mathematical difficulties which may arise in a hollow cylinder containing a crack and also to have an idea on the interaction of the crack and the rigid outer surface for this particular geometry.

\section{Formulation of the problem}

An infinitely long thick-walled cylinder with inner radius A, outer radius B and a ring shaped crack of width $(b-a)$ at the symmetry plane $z=0$, is taken into consideration (see Fig. 1). The crack surfaces are under the action of distributed axisymmetric compressive loads. The inner surface of the cylinder is stress free and the outer wall is rigid.

The governing equations of the axisymmetric elasticity problem in terms of $r$-and $z$-components of the displacement vector, $u(r, z)$ and $w(r, z)$

$$
\begin{aligned}
& (\kappa+1)\left(\frac{\partial^{2} u}{\partial r^{2}}+\frac{1}{r} \frac{\partial u}{\partial r}-\frac{u}{r^{2}}\right)+(\kappa-1) \frac{\partial^{2} u}{\partial z^{2}}+2 \frac{\partial^{2} w}{\partial r \partial z}=0 \\
& 2\left(\frac{\partial^{2} u}{\partial r \partial z}+\frac{1}{r} \frac{\partial u}{\partial z}\right)+(\kappa-1)\left(\frac{\partial^{2} w}{\partial r^{2}}+\frac{1}{r} \frac{\partial w}{\partial r}\right)+(\kappa+1)\left(\frac{\partial^{2} w}{\partial z^{2}}\right)=0
\end{aligned}
$$

must be solved subjected to the following boundary conditions:

$$
\begin{array}{ll}
\sigma_{z}(r, \pm \infty)=0 & (A\langle r<B) \\
w(r, 0)=0 & (A\langle r<a, b\langle r<B), \\
\tau_{r z}(r, 0)=0 & (a\langle r<b), \\
\sigma_{z}(r, 0)=-p(r) & (a\langle r\langle b), \\
\sigma_{r}(A, z)=0 & (-\infty<z<\infty), \\
\tau_{r z}(A, z)=0 & (-\infty<z<\infty), \\
u(B, z)=0 & (-\infty<z<\infty), \\
w(B, z)=0 & (-\infty<z<\infty),
\end{array}
$$

where $\kappa=3-4 v, v$ being the Poisson's ratio, $p(r)$ is the intensity of the known distributed load on the crack surfaces.

The general expressions for the perturbation problem can be obtained as the sum of the general solutions of the following two sub-problems in order to have sufficient number of unknowns in the expressions of the displacements and stresses so that all of the boundary conditions can be satisfied: 


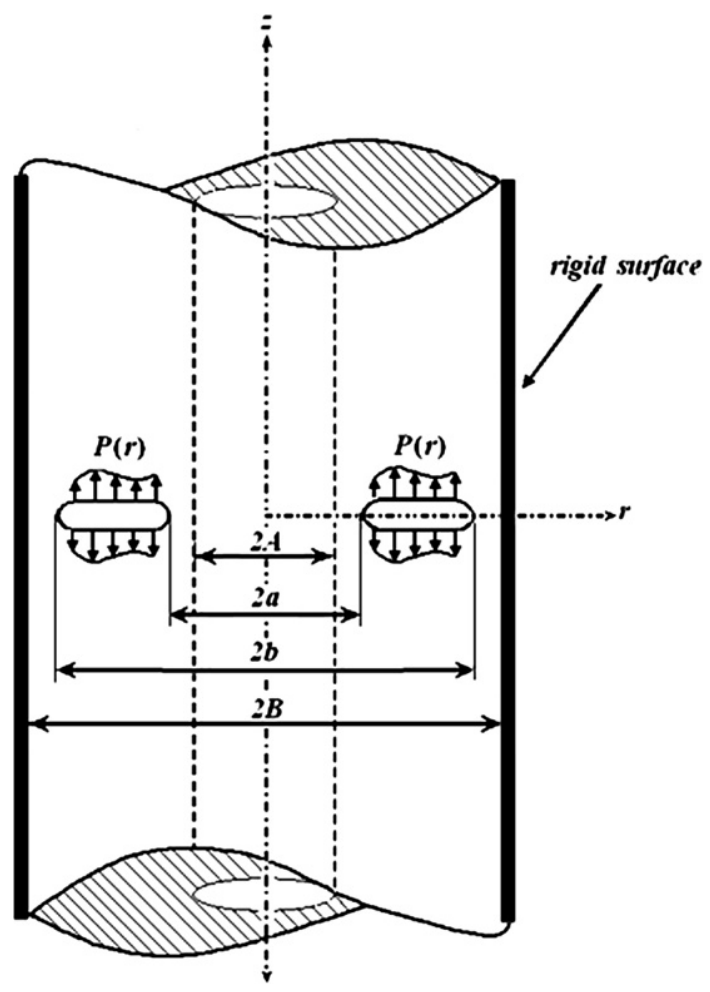

Fig. 1. Geometry of the problem.

(1) Problem of an infinite axisymmetric elastic medium with a ring-shaped crack of width $(b-a)$ at $z=0$ plane. In the solution of Eq. (1), displacements $u(r, z)$ and $w(r, z)$ are assumed to be in the form

$$
\begin{aligned}
& u(r, z)=\int_{0}^{\infty} U(\alpha, z) \alpha J_{1}(\alpha r) \mathrm{d} \alpha, \\
& w(r, z)=\int_{0}^{\infty} W(\alpha, z) \alpha J_{0}(\alpha r) \mathrm{d} \alpha,
\end{aligned}
$$

where $J_{0}$ and $J_{1}$ are the Bessel functions of the first kind of order zero and one, respectively. Substituting (5) and (6) in (1) and inverting the related Hankel transforms, the following system of ordinary differential equations can be obtained

$$
\begin{aligned}
& 2 \alpha \frac{\mathrm{d} U}{\mathrm{~d} z}-\alpha^{2}(\kappa-1) W+(\kappa+1) \frac{\mathrm{d}^{2} W}{\mathrm{~d} z^{2}}=0, \\
& (\kappa+1)\left(-\alpha^{2} U\right)+(\kappa-1) \frac{\mathrm{d}^{2} U}{\mathrm{~d} z^{2}}-2 \alpha \frac{\mathrm{d} W}{\mathrm{~d} z}=0 .
\end{aligned}
$$

The solution of the system of differential equations is found to be

$$
\begin{aligned}
& U(\alpha, z)=\left(c_{1}+c_{2} z\right) \mathrm{e}^{-\alpha z}+\left(c_{3}+c_{4} z\right) \mathrm{e}^{\alpha z}, \\
& W(\alpha, z)=\left[\left(c_{1}+c_{2} z\right)+\frac{\kappa}{z} c_{2}\right] \mathrm{e}^{-\alpha z}+\left[-\left(c_{3}+c_{4} z\right)+\frac{\kappa}{z} c_{4}\right] \mathrm{e}^{\alpha z},
\end{aligned}
$$

where $c_{1}, c_{2}, c_{3}$ and $c_{4}$ are arbitrary unknown constants. 
The axisymmetric infinite medium can be separated into upper and lower half-spaces and the expressions for the displacements and stresses are matched at $z=0$ by considering the following stress type boundary conditions:

$$
\begin{aligned}
& \sigma_{z_{1}}(r, 0)=\sigma_{z_{2}}(r, 0) \quad(0 \leqslant r(\infty), \\
& \tau_{r z_{1}}(r, 0)=\tau_{r z_{2}}(r, 0) \quad(0 \leqslant r(\infty), \\
& \frac{\partial}{\partial r}\left[u_{1}(r, 0)-u_{2}(r, 0)\right]=0 \quad(0 \leqslant r(\infty), \\
& \frac{\partial}{\partial r}\left[w_{1}(r, 0)-w_{2}(r, 0)\right]=2 f(r) \quad(0 \leqslant r(\infty),
\end{aligned}
$$

where $f(r)$ is the unknown crack surface displacement derivative such that $f(r)=0$ when $(A<r<a, b<r<B)$, the subscripts 1 and 2 denote upper and lower half-spaces, respectively.

Since the hollow cylinder having a ring-shaped crack is symmetric about $z=0$ plane, it is sufficient to consider the solution of the axisymmetric problem only in the upper or lower half-space. Therefore, the general expressions for the displacements and the stresses in the upper half-space are found to be [10]:

$$
\begin{aligned}
u_{\text {Hankel }}(r, z)= & \frac{1}{\kappa+1} \int_{0}^{\infty}[-2 \alpha z+(\kappa-1)] F(\alpha) \mathrm{e}^{-\alpha z} J_{1}(\alpha r) \mathrm{d} \alpha, \\
w_{\text {Hankel }}(r, z)= & \frac{1}{\kappa+1} \int_{0}^{\infty}[-2 \alpha z-(\kappa+1)] F(\alpha) \mathrm{e}^{-\alpha z} J_{0}(\alpha r) \mathrm{d} \alpha, \\
\sigma_{r \text { Hankel }}(r, z)= & \frac{2 \mu}{\kappa+1} \int_{0}^{\infty}[2 \alpha z(\kappa-1)] F(\alpha) \mathrm{e}^{-\alpha z} \frac{1}{r} J_{1}(\alpha r) \mathrm{d} \alpha, \\
& +\frac{2 \mu}{\kappa+1} \int_{0}^{\infty} 2(1-\alpha z) F(\alpha) \mathrm{e}^{-\alpha z} \alpha J_{0}(\alpha r) \mathrm{d} \alpha, \\
\sigma_{z \text { Hankel }}(r, z)= & \frac{4 \mu}{\kappa+1} \int_{0}^{\infty}(\alpha z+1) F(\alpha) \mathrm{e}^{-\alpha z} \alpha J_{0}(\alpha r) \mathrm{d} \alpha, \\
\tau_{r z \text { Hankel }}(r, z)= & \frac{4 \mu}{\kappa+1} \int_{0}^{\infty} \alpha z F(\alpha) \mathrm{e}^{-\alpha z} \alpha J_{1}(\alpha r) \mathrm{d} \alpha,
\end{aligned}
$$

where

$$
F(\alpha)=\int_{a}^{b} f(r) r J_{1}(\alpha r) \mathrm{d} r .
$$

and $\mu$ is the shear modulus.

(2) An infinite elastic medium with no crack subjected to arbitrary symmetric loads. The medium is symmetric about both $z$-axis and $z=0$ plane. Governing equations (Eq. (1)) are solved for the displacement components by the use of Fourier sine and cosine transforms in $z$-direction (Watson [11]). Hence, the general expressions for displacements and stresses are obtained as (see [10] for details)

$$
\begin{aligned}
u(r, z)_{\text {Fourier }}= & \frac{2}{\pi} \int_{0}^{\infty}\left[-\frac{1}{2} c_{1} I_{1}(\lambda r)+\frac{1}{2} c_{2} K_{1}(\lambda r)+c_{3} \lambda r I_{0}(\lambda r)+c_{4} \lambda r K_{0}(\lambda r)\right] \cos \lambda z \mathrm{~d} \lambda, \\
w(r, z)_{\text {Fourier }}= & \frac{2}{\pi} \int_{0}^{\infty}\left\{\frac{1}{2} c_{1} I_{0}(\lambda r)+\frac{1}{2} c_{2} K_{0}(\lambda r)-c_{3}\left[(\kappa+1) I_{0}(\lambda r)+\lambda r I_{1}(\lambda r)\right]\right. \\
& \left.+c_{4}\left[(\kappa+1) K_{0}(\lambda r)-\lambda r K_{1}(\lambda r)\right]\right\} \sin \lambda z \mathrm{~d} \lambda, \\
\sigma_{r}(r, z)_{\text {Fourier }}= & \frac{2 \mu}{\pi} \int_{0}^{\infty}\left\{c_{1}\left[-\lambda I_{0}(\lambda r)+\frac{1}{r} I_{1}(\lambda r)\right]+c_{2}\left[-\lambda K_{0}(\lambda r)-\frac{1}{r} K_{1}(\lambda r)\right]\right. \\
& \left.+c_{3}\left[(\kappa-1) \lambda I_{0}(\lambda r)-2 \lambda^{2} r I_{1}(\lambda r)\right]+c_{4}\left[(\kappa-1) \lambda K_{0}(\lambda r)-2 \lambda^{2} r K_{1}(\lambda r)\right]\right\} \cos \lambda z \mathrm{~d} \lambda,
\end{aligned}
$$




$$
\begin{aligned}
\sigma_{z}(r, z)_{\text {Fourier }}= & \frac{2 \mu}{\pi} \int_{0}^{\infty}\left\{c_{1} \lambda I_{0}(\lambda r)+c_{2} \lambda K_{0}(\lambda r)-c_{3}\left[(\kappa+5) \lambda I_{0}(\lambda r)+2 \lambda^{2} r I_{1}(\lambda r)\right]\right. \\
& \left.-c_{4}\left[(\kappa+5) \lambda K_{0}(\lambda r)-2 \lambda^{2} r K_{1}(\lambda r)\right]\right\} \cos \lambda z \mathrm{~d} \lambda, \\
\tau_{r z}(r, z)_{\text {Fourier }}= & \frac{2 \mu}{\pi} \int_{0}^{\infty}\left\{c_{1} \lambda I_{1}(\lambda r)-c_{2} \lambda K_{1}(\lambda r)-c_{3}\left[2 \lambda^{2} r I_{0}(\lambda r)+(\kappa+1) \lambda I_{1}(\lambda r)\right]-c_{4}\left[2 \lambda^{2} r K_{0}(\lambda r)\right.\right. \\
& \left.\left.-(\kappa+1) \lambda K_{1}(\lambda r)\right]\right\} \sin \lambda z \mathrm{~d} \lambda,
\end{aligned}
$$

where $I_{0}, I_{1}$ and $K_{0}, K_{1}$ are the modified Bessel functions of the first and second kinds of order zero and one, respectively.

The expressions for displacements and stresses obtained in Hankel and Fourier solutions of the problem will be added together for the solution of the perturbation problem. After some routine manipulations, arbitrary unknown constants $c_{1}-c_{4}$ appearing in Fourier solution of the problem are obtained in terms of unknown function $F(\alpha)$ using the conditions at inner and outer lateral surfaces of the cylinder (Eqs. (4a)(4d)) as

$$
c_{i}=\left[c_{i 1} S_{3}+c_{i 2} S_{4}+c_{i 3} S_{2}+c_{i 4} S_{1}\right] / D \quad(i=1-4)
$$

where $c_{11}-c_{44}, D$ and $S_{1}-S_{4}$ are given in AppendixA.

\section{Integral equation}

The unknown function $F(\alpha)$ can be determined by the remaining boundary condition on the crack surface stated as follows:

$$
\sigma_{z}(r, 0)=-p(r) \quad(a<r<b) .
$$

Substituting the sum of the expressions given in (11b) and (14b) for the stress in (16), the following singular integral equation with Kernel having Cauchy-type singularity [12] is obtained

$$
\frac{2 \mu}{\pi(\kappa+1)} \int_{a}^{b} f(t)\left[\frac{2}{t-r}+2 M_{1}(r, t)+t N_{11}(r, t)\right] \mathrm{d} t=-p(r) \quad(a\langle r\langle b),
$$

where

$$
\begin{aligned}
& M_{1}(r, t)=\frac{M_{1}^{*}(r, t)-1}{t-r}, \\
& M_{1}^{*}(r, t)= \begin{cases}\frac{2(t-r)}{r} K\left(\frac{t}{r}\right)+\frac{2 r}{t+r} E\left(\frac{t}{r}\right) & (r\rangle t), \\
\frac{2 t}{t+r} E\left(\frac{r}{t}\right) & (r\langle t),\end{cases} \\
& N_{11}(r, t)=\int_{0}^{\infty} L_{11}(r, t, \lambda) \mathrm{d} \lambda,
\end{aligned}
$$

in which $K$ and $E$ are the complete elliptic integrals of the first and the second kinds, respectively.

Eq. (17) must be solved with the single-valuedness condition for the displacement around the crack:

$$
\int_{a}^{b} f(t) \mathrm{d} t=0 .
$$

The integral equation (Eq. (17)) has three types of singularities:

(1) A simple Cauchy-type singularity at $t=r$,

(2) Logarithmic singularity in the Kernel $M_{1}$,

(3) $N_{11}$ has singular terms when $t=A, B$ and $r= \pm A, \pm B$ due to the behavior of the integrand of the integral $N_{11}$ as $\lambda \rightarrow \infty$. Therefore, $N_{11}(r, t)$ can be written in the following form

$$
N_{11}(r, t)=\int_{0}^{\infty} L_{11 \infty}(r, t, \lambda) \mathrm{d} \lambda+\int_{0}^{\infty}\left[L_{11}(r, t, \lambda)-L_{11 \infty}(r, t, \lambda)\right] \mathrm{d} \lambda=N_{11 s}(r, t)+N_{11 b}(r, t)
$$


where

$$
L_{11 \infty}(r, t, \lambda)=\lim _{\lambda \rightarrow \infty} L_{11}(r, t, \lambda) .
$$

Here, $N_{11 s}(r, t)$ and $N_{11 b}(r, t)$ indicate the singular and the bounded parts of the Kernel, respectively.

By using asymptotic expansions for modified Bessel functions of the first and second kinds, $L_{11 \infty}(r, t, \lambda)$ can be obtained in the form

$$
\begin{aligned}
L_{11 \infty}(r, t, \lambda)= & \frac{1}{\sqrt{r t}}\left\{\mathrm{e}^{-\lambda(2 B-r-t)}\left[\frac{1}{\kappa}\left(-4(B-r)(B-t) \lambda^{2}-2(B-r) \lambda+6(B-t) \lambda+\left(\kappa^{2}+3\right)\right)\right]\right. \\
& \left.+\mathrm{e}^{\lambda(2 A-r-t)}\left[4(A-r)(A-t) \lambda^{2}+2(A-r) \lambda+6(A-t) \lambda+4\right]\right\} .
\end{aligned}
$$

The singular part of the Kernel can be obtained by integrating $L_{11 \infty}(r, t, \lambda)$ as

$$
\begin{aligned}
N_{11 s}(r, t)= & \frac{1}{\sqrt{r t}}\left\{\left[\frac{1}{\kappa}\left(-4(B-r)^{2} \frac{\mathrm{d}^{2}}{\mathrm{~d} r^{2}}-12(B-r) \frac{\mathrm{d}}{\mathrm{d} r}+\left(3-\kappa^{2}\right)\right)\right] \frac{1}{(t+r-2 B)}\right. \\
& \left.+\left[-4(A-r) \frac{\mathrm{d}^{2}}{\mathrm{~d} r^{2}}+12(A-r) \frac{\mathrm{d}}{\mathrm{d} r}-2\right] \frac{1}{(t+r-2 A)}\right\} .
\end{aligned}
$$

The unknown function $f(t)$ is expected to be singular at the edges of the crack $r=a, r=\mathrm{b}$ and therefore its behavior may be determined by writing

$$
f(t)=F^{*}(t)[(t-a)(b-t)]^{-\gamma} \quad(0\langle\operatorname{Re}(\gamma)\langle 1),
$$

where $F^{*}(t)$ is Hölder-continuous function in the interval $[a, b]$ and $\gamma$ is an unknown constant. $f(t)$ has an integrable singularity at the edges of the crack. Evaluating the integral containing the singular term using the technique given in [12] and using the procedure described in Artem [13], the following characteristic equation for $\gamma$ can be obtained:

$$
\cot \pi \gamma=0 \text {. }
$$

Therefore $\gamma=1 / 2$ which is in perfect agreement with previously reported results for the power of stress singularity at the tips of an imbedded crack.

\section{Solution of integral equation}

Having determined the singular behavior of the unknown function, the integral appearing in Eq. (17) may be non-dimensionalized for convenience in the numerical scheme introducing the following dimensionless variables $\tau, \xi$ on the crack by

$$
\begin{aligned}
& t=\frac{b-a}{2} \tau+\frac{b+a}{2} \quad(a\langle t\langle b,-1<\tau<1), \\
& r=\frac{b-a}{2} \xi+\frac{b+a}{2} \quad(a\langle r\langle b,-1<\xi<1) .
\end{aligned}
$$

After some manipulations Eq. (17) becomes

$$
\frac{1}{\pi} \int_{-1}^{1} \bar{f}(\tau)\left[\frac{2}{\tau-\xi}+\widehat{M}_{1}(\xi, \tau)+\widehat{N}_{11}(\xi, \tau)\right] \mathrm{d} \tau=-\frac{p(\xi)(\kappa+1)}{2 \mu} \quad(-1\langle\xi\langle 1),
$$


where

$$
\begin{aligned}
& \bar{f}(\tau)=f\left(\frac{b-a}{2} \tau+\frac{b+a}{2}\right), \quad p(\xi)=p\left(\frac{b-a}{2} \xi+\frac{b+a}{2}\right) \\
& \widehat{M}_{1}(\xi, \tau)=(b-a) M_{1}(\xi, \tau), \\
& \widehat{N}_{11}(\xi, \tau)=\left(\frac{b-a}{2}\right)\left(\frac{b-a}{2} \tau+\frac{b-a}{2}\right) N_{11}(\xi, \tau) .
\end{aligned}
$$

Then, substituting singular behavior of the dimensionless unknown function

$$
\bar{f}(\tau)=\bar{F}^{*}(\tau)\left(1-\tau^{2}\right)^{-1 / 2},
$$

in Eq. (30), the singular integral equation becomes:

$$
\frac{1}{\pi} \int_{-1}^{1} \frac{\bar{F}^{*}(\tau)}{\sqrt{1-\tau^{2}}}\left[\frac{2}{\tau-\xi}+\widehat{M}_{1}(\xi, \tau)+\widehat{N}_{11}(\xi, \tau)\right] \mathrm{d} \tau=-\frac{p(\xi)(\kappa+1)}{2 \mu} \quad(-1\langle\xi\langle 1),
$$

where

$$
\bar{F}^{*}(\tau)=F^{*}\left(\frac{b-a}{2} \tau+\frac{b+a}{2}\right)\left(\frac{b-a}{2}\right)^{-1} .
$$

By using the Gauss-Lobatto integration formula [14], the integral equation can be reduced to the following system of algebraic equations:

$$
\sum_{i=1}^{n} C_{i} \bar{F}^{*}\left(\tau_{i}\right)\left[\frac{2}{\tau_{i}-\xi_{i}}+\widehat{M}_{1}\left(\xi_{i}, \tau_{i}\right)+\widehat{N}_{11}\left(\xi_{i}, \tau_{i}\right)\right] \mathrm{d} \tau=-\frac{p(\xi)(\kappa+1)}{2 \mu},
$$

where

$$
\begin{aligned}
\tau_{i} & =\cos [(i-1) \pi /(n-1)] \quad(i=1,2, \ldots, n), \\
\xi_{j} & =\cos [(2 j-1) \pi / 2(n-1)] \quad(j=1,2, \ldots, n-1),
\end{aligned}
$$

are the roots and the weighting constants of related Lobatto polynomials are

$$
C_{1}=C_{n}=\frac{1}{2(n-1)}, \quad C_{i}=\frac{1}{n-1} \quad(i=2,3, \ldots, n-1)
$$

Eq. (37) contains $(n-1)$ equations for $n$-unknowns, $\bar{F}^{*}\left(\tau_{i}\right)$. Since the number of unknowns is larger than the number of equations, the single-valuedness condition

$$
\sum_{i=1}^{n} C_{i} \bar{F}^{*}\left(\tau_{i}\right)=0 \quad(-1<\tau<1)
$$

must be taken into consideration to complete the system to $n$ equations.

\section{Results and discussion}

The system of algebraic equations is solved numerically for unknowns $\bar{F}^{*}\left(\tau_{i}\right)$ at discrete collocation points. The infinite integral appearing in the bounded part of $N_{11}(\xi, \tau)$ in Eq. (37) may be evaluated by using Laguerre quadrature formula given in Stroud and Secrest [14]. After the numerical solution is obtained one can calculate the stress state at the close vicinity of the points $(r=a$ and $r=b$ at $z=0)$ described by means of so-called Mode I stress intensity factors at the edges of the crack

$$
\begin{aligned}
& k_{1}(a)=\lim _{r \rightarrow a} \sqrt{2(a-r)} \sigma_{z}(r, 0), \\
& k_{1}(b)=\lim _{r \rightarrow b} \sqrt{2(r-b)} \sigma_{z}(r, 0),
\end{aligned}
$$


or

$$
\begin{aligned}
& \bar{k}_{1}(a)=k_{1}(a) / p(r) \sqrt{(b-a) / 2}=\frac{2}{s(\kappa+1)} \bar{F}^{*}(-1), \\
& \bar{k}_{1}(b)=k_{1}(b) / p(r) \sqrt{(b-a) / 2}=-\frac{2}{s(\kappa+1)} \bar{F}^{*}(1),
\end{aligned}
$$

as normalized stress intensity factors. Here, $s=p(r) / 2 \mu$. Normalized stress intensity factors $\bar{k}_{1}(a)$ and $\bar{k}_{1}(b)$ are calculated for various geometric configurations for different values of Poisson's ratio as the material parameter. As examples, results are obtained for the following load distributions on crack surfaces:

$$
\begin{aligned}
& p_{0}(r)=p_{0} \\
& p_{1}(r)=\frac{3\left(b^{2}-a^{2}\right)(r-B)}{2 b^{3}-3 B b^{2}-2 a^{3}+3 B a^{2}} p_{0} \\
& p_{2}(r)=\frac{6\left(b^{2}-a^{2}\right)(r-B)^{2}}{3 b^{4}-8 B b^{3}+6 B^{2} b^{2}-3 a^{4}+8 B a^{3}-6 B^{2} a^{2}} p_{0}
\end{aligned}
$$

where $p_{0}$ is the mean of compressive distributed load on crack surfaces. The uniform pressure on crack surfaces is considered for the purpose of possible comparisons, since extensive number of examples with uniform load appear in the literature. The outer lateral surface of the cylinder is rigidly fixed while the inner surface is free. It is obvious that the stress distribution at the location of the crack for an infinite cylinder loaded at infinity (even with uniform axial loads) will not be uniform. It will rather be varying with the radial coordinate $r$. In order to present additional useful results one may expect for the perturbation problem in such situations where the infinite cylinder is loaded at infinity, here linearly varying and parabolically varying load distributions on the crack surfaces are also considered.

Defining the dimensionless parameters

$$
r_{1}=\frac{A}{B}, \quad r_{2}=\frac{a}{B}, \quad r_{3}=\frac{b}{B}
$$

the following dimensionless distributions are obtained

$$
\begin{aligned}
& \bar{p}_{0}(\xi)=p_{0} \\
& \bar{p}_{1}(\xi)=\frac{3\left(r_{3}^{2}-r_{2}^{2}\right)\left(\left(r_{3}-r_{2}\right) \xi+r_{3}+r_{2}-2\right)}{2\left(2 r_{3}^{3}-3 r_{3}^{2}-2 r_{2}^{3}+3 r_{2}^{2}\right)} p_{0} \\
& \bar{p}_{2}(\xi)=\frac{3\left(r_{3}^{2}-r_{2}^{2}\right)\left(\left(r_{3}-r_{2}\right) \xi+r_{3}+r_{2}-2\right)^{2}}{2\left(3 r_{3}^{4}-8 r_{3}^{3}+6 r_{3}^{2}-3 r_{2}^{4}+8 r_{2}^{3}-6 r_{2}^{2}\right)} p_{0}
\end{aligned}
$$

As a starting point to check the formulation and the numerical scheme of the problem we consider the following case: when the crack size becomes very small compared to other dimensions of the cylinder (say, $(b-a)$ / $B=10^{-5}$ ), the problem turns out to be a finite crack in an infinite medium and therefore normalized stress intensity factors at the edges of the crack, $\bar{k}_{1}(a)$ and $\bar{k}_{1}(b)$, approach unity. This is the well-known result for crack tips surrounded by a homogenous medium.

Fig. 2 shows the variation of normalized Mode I stress intensity factors at the edges of the internal central crack (the thickness of the net ligaments, $(a-A)$ and $(B-b)$, are equal) for different loading conditions. As the thickness of the net ligaments decreases, in other words, as the crack size increases, the normalized stress intensity factors at the inner edge of the crack, $\bar{k}_{1}(a)$, increases while at the outer edge of the crack, $\bar{k}_{1}(b)$, decreases. From Table 1, it can also be observed that both $\bar{k}_{1}(a)$ and $\bar{k}_{1}(b)$ decrease as the wall thickness of cylinder increases. Results for $\bar{k}_{1}(a)$ and $\bar{k}_{1}(b)$ for different load distributions with $r_{1}=A / B=0.25$ and $r_{3}=b / B=0.8$ are also given for comparison in Fig. 3. It can be observed from Fig. 4 that $\bar{k}_{1}(a)$ increases 


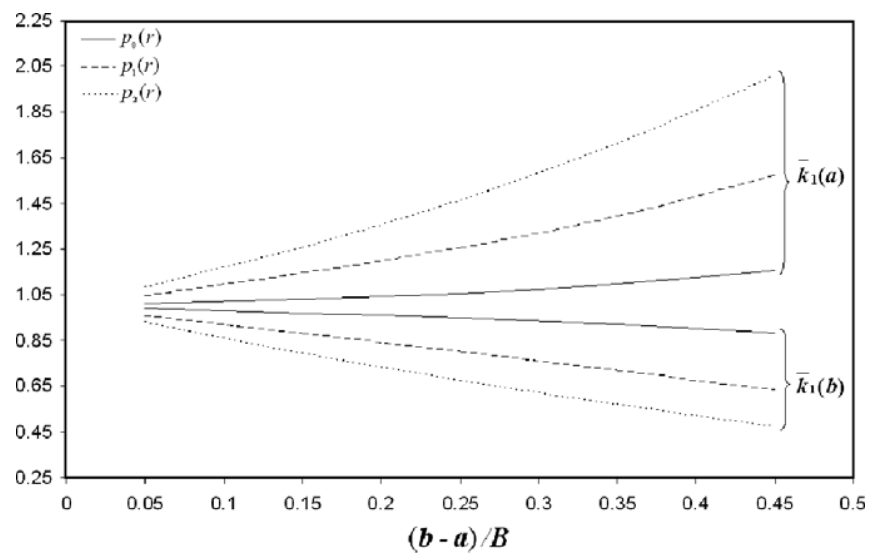

Fig. 2. Variation of Mode I normalized stress intensity factors $\bar{k}_{1}(a)$ and $\bar{k}_{1}(b)$ for internal central crack with different load distributions $(A / B=0.25)$.

Table 1

Variation of normalized stress intensity factors, $\bar{k}_{1}(a)$ and $\bar{k}_{1}(b)$, for the internal central crack in the thick-walled cylinder $\left(\bar{k}_{1}(a)=k_{1}(a) / p_{0} \sqrt{(b-a) / 2}, \bar{k}_{1}(b)=k_{1}(b) / p_{0} \sqrt{(b-a) / 2}, v=0.3\right)$

\begin{tabular}{|c|c|c|c|c|c|c|}
\hline \multirow{2}{*}{$\frac{b-a}{B}$} & \multicolumn{3}{|l|}{$\bar{k}_{1}(a)$} & \multicolumn{3}{|l|}{$\bar{k}_{1}(b)$} \\
\hline & $A / B=0.25$ & $A / B=0.20$ & $A / B=0.125$ & $A / B=0.25$ & $A / B=0.20$ & $A / B=0.125$ \\
\hline$\rightarrow 0$ & $\rightarrow 1.0$ & $\rightarrow 1.0$ & $\rightarrow 1.0$ & $\rightarrow 1.0$ & $\rightarrow 1.0$ & $\rightarrow 1.0$ \\
\hline 0.05 & 1.00999 & 1.01011 & 1.01067 & 0.990367 & 0.989731 & 0.988878 \\
\hline 0.10 & 1.02038 & 1.01990 & 1.02083 & 0.980565 & 0.978931 & 0.976687 \\
\hline 0.15 & 1.03145 & 1.02944 & 1.03053 & 0.970255 & 0.967435 & 0.962990 \\
\hline 0.20 & 1.04367 & 1.03902 & 1.03989 & 0.959180 & 0.955179 & 0.947453 \\
\hline 0.25 & 1.05775 & 1.04918 & 1.04905 & 0.947087 & 0.942099 & 0.929777 \\
\hline 0.30 & 1.07464 & 1.06079 & 1.05815 & 0.933708 & 0.928073 & 0.909688 \\
\hline 0.35 & 1.09547 & 1.07516 & 1.06727 & 0.918781 & 0.912862 & 0.886957 \\
\hline 0.40 & 1.12141 & 1.09415 & 1.07641 & 0.902146 & 0.896074 & 0.861424 \\
\hline 0.45 & 1.15331 & 1.12023 & 1.08542 & 0.883965 & 0.877178 & 0.833042 \\
\hline 0.50 & 1.19078 & 1.15639 & 1.09406 & 0.865168 & 0.855651 & 0.801900 \\
\hline
\end{tabular}

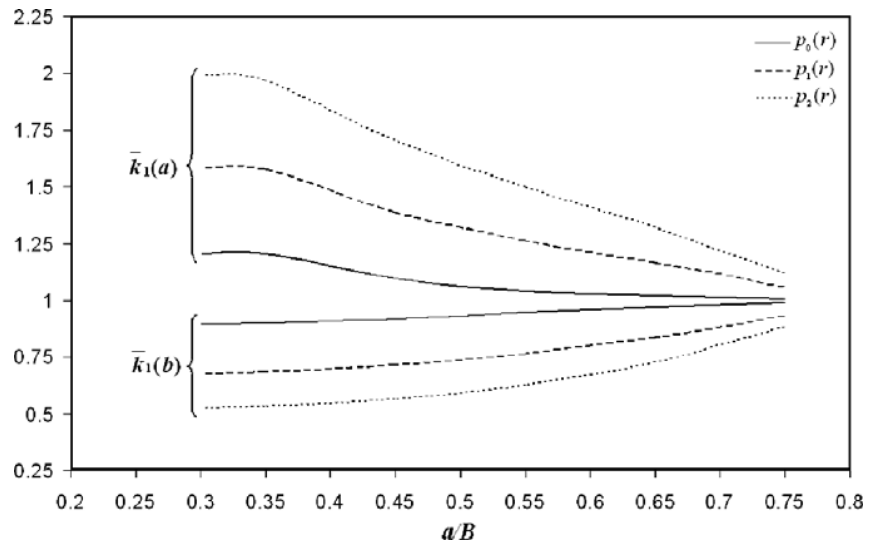

Fig. 3. Variation of Mode I normalized stress intensity factors $\bar{k}_{1}(a)$ and $\bar{k}_{1}(b)$ with $a / B$ for different load distributions $(A / B=0.25, b /$ $B=0.8, v=0.3$ ). 


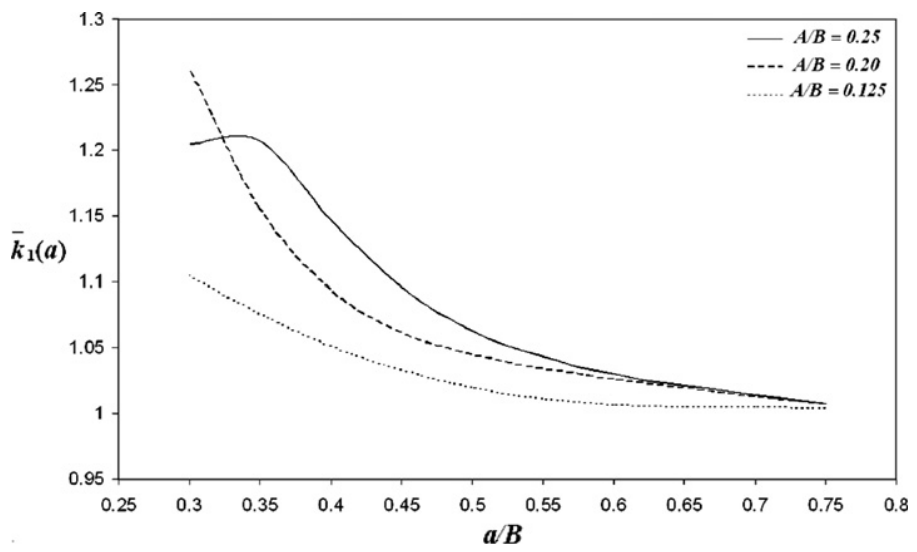

Fig. 4. Variation of Mode I normalized stress intensity factor $\bar{k}_{1}(a)$ with $a / B$ for different $A / B$ values $(b / B=0.8, v=0.3)$.

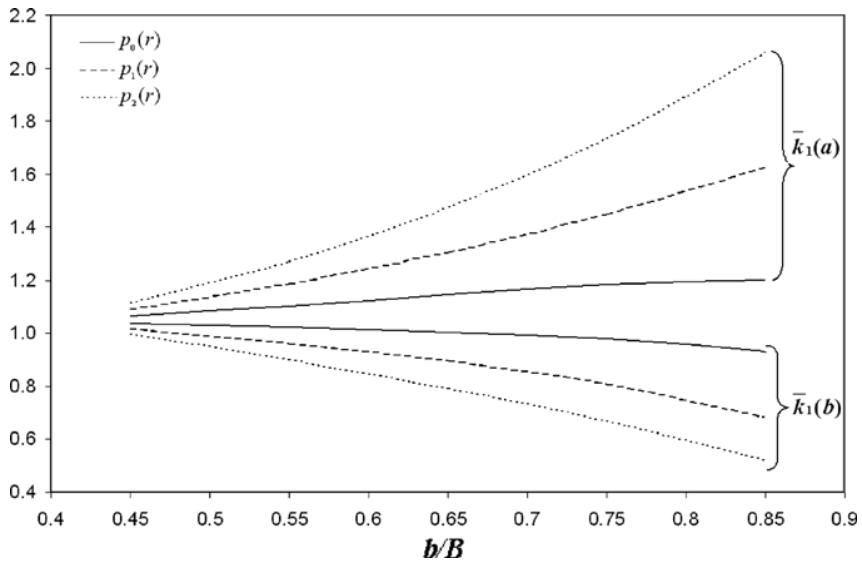

Fig. 5. Variation of Mode I normalized stress intensity factors $\bar{k}_{1}(a)$ and $\bar{k}_{1}(b)$ with $b / B$ for different load distributions $(A / B=0.25, a /$ $B=0.4, v=0.3)$.

as $a / B$ decreases, but only when $a / B$ decreases from 0.35 to 0.30 for $r_{1}=A / B=0.25$, there is a slight decrease. In this case there is a considerable interaction between the crack and the stress-free inner surface of the cylinder. The Kernel used in the present study is no longer bounded in the corresponding closed interval, and, of course, the single-valuedness condition is no longer valid [1]. However for the cases $r_{1}=A / B=0.125$ and $r_{1}=A / B=0.2$, a similar behavior is not observed because the crack is far enough from the inner surface of the cylinder.

Fig. 5 shows variation of $\bar{k}_{1}(a)$ and $\bar{k}_{1}(b)$, normalized Mode I stress intensity factors at the edges of the crack, for $r_{1}=A / B=0.25$ with $r_{2}=a / B=0.4$. As it is expected, $\bar{k}_{1}(b)$ decreases when $b / B$ increases. This means that the rigid outer surface prevents the crack from opening. In other words, the strength of stress singularity decreases.

Another observation is that for fixed $(b-a)$, when $a / B$ increases, that is, when the crack approaches the rigid surface, as it is expected, both $\bar{k}_{1}(a)$ and $\bar{k}_{1}(b)$ decrease. The behavior of $\bar{k}_{1}(a)$ and $\bar{k}_{1}(b)$ for different crack lengths and the uniform load $p_{0}$ only, are shown in Fig. 6.

Normalized stress intensity factors $\bar{k}_{1}(a)$ and $\bar{k}_{1}(b)$, are also calculated for different values of Poisson's ratio and the results are illustrated in Fig. 7. 


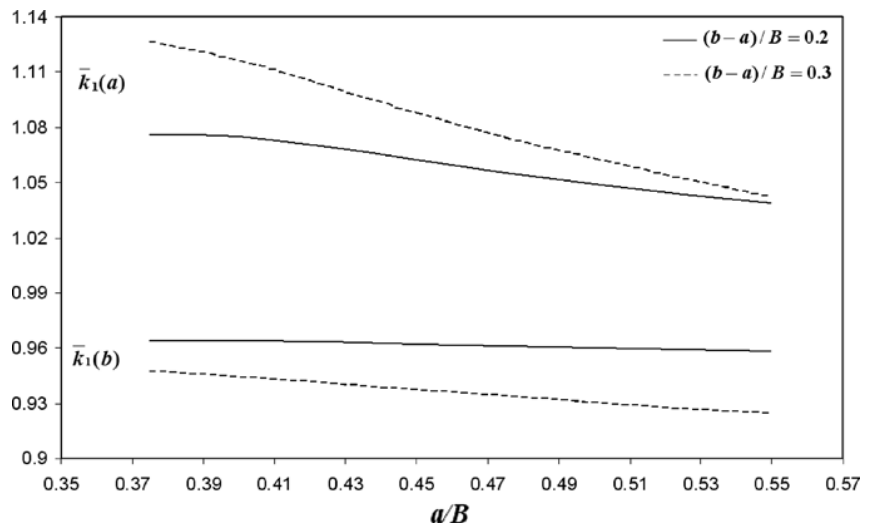

Fig. 6. Variation of Mode I normalized stress intensity factors $\bar{k}_{1}(a)$ and $\bar{k}_{1}(b)$ with $a / B$ for two different fixed crack lengths.

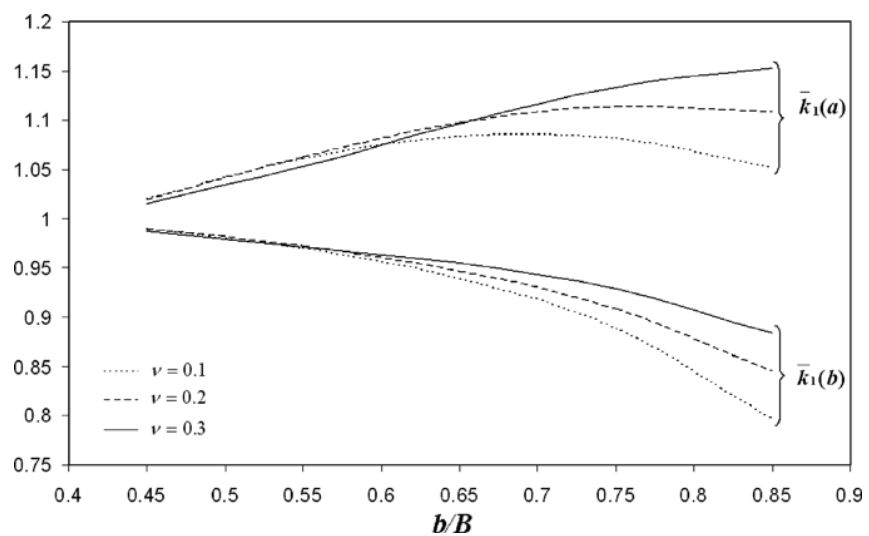

Fig. 7. Variation of Mode I normalized stress intensity factors $\bar{k}_{1}(a)$ and $\bar{k}_{1}(b)$ with $b / B$ for different values of Poisson's ratio $(A / B=0.25)$.

\section{Conclusions}

The present paper considers the investigation of the stress intensity factors at the edges of the crack for a long thick-walled cylinder containing a ring-shaped crack whose surfaces are subjected to compressive distributed load. The normalized Mode I stress intensity factors are computed for the crack and the results are presented in graphical and tabular forms. It is observed that $\bar{k}_{1}(a)$ is always greater than $\bar{k}_{1}(b)$. The influence of rigid surface is discussed in this study. It is concluded that as the crack approaches the rigid surface, normalized Mode I stress intensity factor at the outer edge of the crack decreases. This is because the rigid wall prevents the crack from opening. In other words, the strength of stress singularity decreases. Comparing the results for different loadings, we see that they show similar behavior. Finally, it is also observed that the stress intensity factors are affected by the Poisson's ratio as material property.

\section{Acknowledgement}

Fruitful discussions about the present study with Professor M.R. Gecit of the Middle East Technical University are gratefully acknowledged.

\section{Appendix A}

The coefficients which appear in Eq. (15) are 


$$
\begin{aligned}
& c_{11}=\left(A B\left(K_{0}(B \lambda) H_{00}+K_{1}(B \lambda) H_{01}\right)-\frac{B K_{1}(A \lambda)}{\lambda}\right) \lambda^{3} \\
& +\left(A(\kappa+1) K_{1}(B \lambda) H_{00}-\frac{1}{2} B(\kappa+1)\left(K_{0}(B \lambda) H_{10}+K_{1}(B \lambda) H_{11}\right) \lambda^{2}+\frac{1}{2}(\kappa+1)^{2} K_{1}(B \lambda) H_{10} \lambda\right. \\
& c_{12}=\left(\frac{B K_{0}(A \lambda)}{\lambda}-A B\left(K_{0}(B \lambda) H_{10}+K_{1}(B \lambda) H_{11}\right)\right) \lambda^{3} \\
& +\left(\frac{B K_{1}(A \lambda)}{A \lambda}+\frac{1}{2} B(\kappa-1)\left(K_{0}(B \lambda) H_{10}+K_{1}(B \lambda) H_{01}\right)-A(\kappa+1) K_{1}(B \lambda) H_{10}\right) \lambda^{2} \\
& +\frac{1}{2}\left(\kappa^{2}-1\right) K_{1}(B \lambda) H_{00} \lambda \\
& c_{13}=\left(2 A B\left(H_{00} K_{0}(A \lambda)-H_{10} K_{1}(A \lambda)+\frac{2 A K_{1}(B \lambda)}{\lambda}\right) \lambda^{4}-\frac{B(\kappa+1) K_{0}(B \lambda) \lambda^{2}}{A}\right. \\
& +\left(\frac{\left(1-\kappa^{2}\right) K_{1}(B \lambda)}{2 A \lambda}-\frac{B H_{10}(\kappa+1) K_{1}(A \lambda)}{A}\right) \lambda^{2} \\
& c_{14}=\left(2 A B\left(H_{01} K_{0}(A \lambda)-H_{11} K_{1}(A \lambda)-\frac{2 A K_{0}(B \lambda)}{\lambda}\right) \lambda^{4}\right. \\
& +\left(2 A(\kappa+1)\left(H_{00} K_{0}(A \lambda)-H_{10} K_{1}(A \lambda)\right)+\frac{B(\kappa+1) K_{1}(B \lambda)}{A \lambda}\right) \lambda^{3} \\
& +\left(-\frac{(\kappa+1)(\kappa+3) K_{0}(B \lambda)}{2 A \lambda}-\frac{B H_{11}(\kappa+1) K_{1}(A \lambda)}{A}\right) \lambda^{2}-\frac{H_{10}(\kappa+1)^{2} K_{1}(A \lambda) \lambda}{A} \\
& c_{21}=\left(-A B H_{00} I_{0}(B \lambda)-\frac{B I_{1}(A \lambda)}{\lambda}+A B H_{01} I_{1}(B \lambda)\right) \lambda^{3} \\
& +\left(\frac{1}{2} B H_{10}(\kappa+1) I_{0}(B \lambda)+A H_{00}(\kappa+1) I_{1}(B \lambda)-\frac{1}{2} B H_{11}(\kappa+1) I_{1}(B \lambda)\right) \lambda^{2}-\frac{1}{2} B H_{10}(\kappa+1) I_{1}(B \lambda) \lambda \\
& c_{22}=\left(-\frac{B I_{0}(A \lambda)}{\lambda}+A B H_{10} I_{0}(B \lambda)-A B H_{11} I_{1}(B \lambda)\right) \lambda^{3}+\frac{1}{2} H_{00}\left(\kappa^{2}-1\right) I_{1}(B \lambda) \lambda \\
& +\left(-\frac{1}{2} B H_{00}(\kappa-1) I_{0}(B \lambda)+\frac{B I_{1}(A \lambda)}{A \lambda}+\frac{1}{2} B H_{01}(\kappa-1) I_{1}(B \lambda)-A H_{10}(\kappa+1) I_{1}(B \lambda)\right) \lambda^{2} \\
& c_{23}=\left(-2 A B H_{00} I_{0}(A \lambda)-2 A B H_{10} I_{1}(A \lambda)+\frac{2 A I_{1}(B \lambda)}{\lambda}\right) \lambda^{4}+\frac{B(\kappa+1) I_{0}(B \lambda) \lambda^{2}}{A} \\
& +\left(-\frac{B H_{10} I_{1}(A \lambda)}{A}-\frac{B H_{10} \kappa I_{1}(A \lambda)}{A}+\frac{\left(1-\kappa^{2}\right) I_{1}(B \lambda)}{2 A \lambda}\right) \lambda^{2} \\
& c_{24}=\left(-2 A B H_{01} I_{0}(A \lambda)+\frac{2 A I_{0}(B \lambda)}{\lambda}-2 A B H_{11} I_{1}(A \lambda)\right) \lambda^{4} \\
& +\left(-2 A H_{00} I_{0}(A \lambda)-2 A H_{00} \kappa I_{0}(A \lambda)-2 A H_{10} I_{1}(A \lambda)-2 A H_{10} \kappa I_{1}(A \lambda)\right. \\
& \left.+\frac{B I_{1}(B \lambda)}{A \lambda}+\frac{B \kappa I_{1}(B \lambda)}{A \lambda}\right) \lambda^{3}+\left(\frac{\left(\kappa^{2}+4 \kappa+3\right) I_{0}(B \lambda)}{2 A \lambda}-\frac{B H_{11} I_{1}(A \lambda)}{A}\right) \lambda^{2}-\frac{H_{10}(\kappa+1)^{2} I_{1}(A \lambda) \lambda}{A} \\
& c_{31}=\left(\frac{A K_{0}(A \lambda)}{2 B \lambda}-\frac{1}{2} B H_{10} K_{0}(B \lambda)-\frac{1}{2} B H_{11} K_{1}(B \lambda)\right) \lambda^{2}+\frac{1}{4}(\kappa+1)\left(H_{11} K_{0}(B \lambda)-H_{10} K_{1}(B \lambda)\right) \lambda
\end{aligned}
$$




$$
\begin{aligned}
& c_{32}=\left(\frac{1}{2} B H_{00} K_{0}(B \lambda)-\frac{A K_{1}(A \lambda)}{2 B \lambda}+\frac{1}{2} B H_{01} K_{1}(B \lambda)\right) \lambda^{2} \\
& +\left(-\frac{K_{0}(A \lambda)}{4 B \lambda}-\frac{1}{2} H_{01} K_{0}(B \lambda)+\frac{B H_{10} K_{0}(B \lambda)}{2 A}-\frac{1}{4} H_{01} \kappa K_{0}(B \lambda)+\frac{B H_{11} K_{1}(B \lambda)}{2 A}+\frac{1}{4} H_{00} \kappa K_{1}(B \lambda)\right) \lambda \\
& -\frac{H_{11}(\kappa+1) K_{0}(B \lambda)}{2 A} \\
& c_{33}=\left(A H_{01} K_{0}(A \lambda)-\frac{B K_{0}(B \lambda)}{A \lambda}-A H_{11} K_{1}(A \lambda)\right) \lambda^{3}-\frac{H_{11}(\kappa+1) K_{1}(A \lambda) \lambda}{2 A}-\frac{(\kappa+1) K_{1}(B \lambda) \lambda}{2 A} \\
& c_{34}=\left(A H_{00} K_{0}(A \lambda)-A H_{10} K_{1}(A \lambda)+\frac{B K_{1}(B \lambda)}{A \lambda}\right) \lambda^{3}-\frac{(\kappa+1) K_{0}(B \lambda) \lambda}{2 A}-\frac{H_{10}(\kappa+1) K_{1}(A \lambda) \lambda}{2 A} \\
& c_{41}=\left(-\frac{A I_{0}(A \lambda)}{2 B \lambda}+\frac{1}{2} B H_{10} I_{0}(B \lambda)-\frac{1}{2} B H_{11} I_{1}(B \lambda)\right) \lambda^{2}+\frac{1}{4}(-\kappa-1)\left(H_{11} I_{0}(B \lambda)+H_{10} I_{1}(B \lambda)\right) \lambda \\
& c_{42}=\left(-\frac{1}{2} B H_{00} I_{0}(B \lambda)-\frac{A I_{1}(A \lambda)}{2 B \lambda}+\frac{1}{2} B H_{01} I_{1}(B \lambda)\right) \lambda^{2} \\
& +\left(\frac{I_{0}(A \lambda)}{4 B \lambda}+\frac{1}{2} H_{01} I_{0}(B \lambda)-\frac{B H_{10} I_{0}(B \lambda)}{2 A}+\frac{1}{4} H_{01} \kappa I_{0}(B \lambda)+\frac{B H_{11} I_{1}(B \lambda)}{2 A}+\frac{1}{4} H_{00} \kappa I_{1}(B \lambda)\right) \lambda \\
& +\frac{H_{11}(\kappa+1) I_{0}(B \lambda)}{2 A} \\
& c_{43}=\left(-A H_{01} I_{0}(A \lambda)+\frac{B I_{0}(B \lambda)}{A \lambda}-A H_{11} I_{1}(A \lambda)\right) \lambda^{3}-\frac{H_{11}(\kappa+1) I_{1}(A \lambda) \lambda}{2 A}-\frac{I_{1}(B \lambda) \lambda}{2 A} \\
& c_{44}=\left(-A H_{00} I_{0}(A \lambda)-A H_{10} I_{1}(A \lambda)+\frac{B I_{1}(B \lambda)}{A \lambda}\right) \lambda^{3}-\frac{(\kappa+1) I_{0}(B \lambda) \lambda}{2 A}-\frac{H_{10}(\kappa+1) I_{1}(A \lambda) \lambda}{2 A} \\
& D=A B I_{0}^{2}(B \lambda) K_{0}^{2}(A \lambda) \lambda^{4}-A B I_{1}^{2}(B \lambda) K_{0}^{2}(A \lambda) \lambda^{4}+A B I_{0}^{2}(A \lambda) K_{0}^{2}(B \lambda) \lambda^{4} \\
& -A B I_{1}^{2}(A \lambda) K_{0}^{2}(B \lambda) \lambda^{4}-A B I_{0}^{2}(B \lambda) K_{1}^{2}(A \lambda) \lambda^{4}+A B I_{1}^{2}(B \lambda) K_{1}^{2}(A \lambda) \lambda^{4} \\
& -2 A B I_{0}(B \lambda) I_{1}(A \lambda) K_{0}(B \lambda) K_{1}(A \lambda) \lambda^{4}-2 A B I_{0}(A \lambda) I_{1}(B \lambda) K_{0}(A \lambda) K_{1}(B \lambda) \lambda^{4} \\
& -2 A B I_{1}(A \lambda) I_{1}(B \lambda) K_{1}(A \lambda) K_{1}(B \lambda) \lambda^{4}-A I_{0}(B \lambda) I_{1}(B \lambda) K_{0}^{2}(A \lambda) \lambda^{3} \\
& -A \kappa I_{0}(B \lambda) I_{1}(B \lambda) K_{0}^{2}(A \lambda) \lambda^{3}+A \kappa I_{0}(B \lambda) I_{1}(B \lambda) K_{1}^{2}(A \lambda) \lambda^{3} \\
& +A I_{0}(A \lambda) I_{1}(B \lambda) K_{0}(A \lambda) K_{0}(B \lambda) \lambda^{3}+A \kappa I_{0}(A \lambda) I_{1}(B \lambda) K_{0}(A \lambda) K_{0}(B \lambda) \lambda^{3} \\
& \text { - } A I_{1}(A \lambda) I_{1}(B \lambda) K_{0}(A \lambda) K_{0}(B \lambda) \lambda^{3}-A I_{0}(B \lambda) I_{1}(B \lambda) K_{0}(A \lambda) K_{1}(A \lambda) \lambda^{3} \\
& +A \kappa I_{1}(A \lambda) I_{1}(B \lambda) K_{0}(B \lambda) K_{1}(A \lambda) \lambda^{3}+A I_{0}(A \lambda) I_{0}(B \lambda) K_{0}(A \lambda) K_{1}(B \lambda) \lambda^{3} \\
& -A \kappa I_{0}(A \lambda) I_{0}(B \lambda) K_{0}(A \lambda) K_{1}(B \lambda) \lambda^{3}-A I_{0}^{2}(A \lambda) K_{0}(B \lambda) K_{1}(B \lambda) \lambda^{3} \\
& +A \kappa I_{0}^{2}(A \lambda) K_{0}(B \lambda) K_{1}(B \lambda) \lambda^{3}-A I_{0}(A \lambda) I_{0}(B \lambda) K_{0}(A \lambda) K_{1}(B \lambda) \lambda^{3} \\
& +A \kappa I_{1}^{2}(A \lambda) K_{0}(B \lambda) K_{1}(B \lambda) \lambda^{3}+A I_{0}(A \lambda) I_{1}(A \lambda) K_{0}(B \lambda) K_{1}(B \lambda) \lambda^{3} \\
& +A I_{0}(A \lambda) I_{0}(B \lambda) K_{1}(A \lambda) K_{1}(B \lambda) \lambda^{3}-A \kappa I_{0}(B \lambda) I_{1}(A \lambda) K_{1}(A \lambda) K_{1}(B \lambda) \lambda^{3} \\
& -\frac{B I_{1}^{2}(A \lambda) K_{0}^{2}(B \lambda) \lambda^{2}}{2 A}-\frac{B \kappa I_{1}^{2}(A \lambda) K_{0}^{2}(B \lambda) \lambda^{2}}{2 A}-\frac{B I_{0}^{2}(B \lambda) K_{1}^{2}(A \lambda) \lambda^{2}}{2 A} \\
& -\frac{B \kappa I_{0}^{2}(B \lambda) K_{1}^{2}(A \lambda) \lambda^{2}}{2 A}+\frac{B I_{1}^{2}(B \lambda) K_{1}^{2}(A \lambda) \lambda^{2}}{2 A}+\frac{B \kappa I_{1}^{2}(B \lambda) K_{1}^{2}(A \lambda) \lambda^{2}}{2 A} \\
& +\frac{B I_{1}^{2}(A \lambda) K_{1}^{2}(B \lambda) \lambda^{2}}{2 A}+\frac{B \kappa I_{1}^{2}(A \lambda) K_{1}^{2}(B \lambda) \lambda^{2}}{2 A}+\frac{B \lambda^{2}}{A}
\end{aligned}
$$




$$
\begin{aligned}
- & \frac{B I_{0}(B \lambda) I_{1}(A \lambda) K_{0}(B \lambda) K_{1}(A \lambda) \lambda^{2}}{A}+\frac{\kappa^{2} I_{1}(A \lambda) I_{1}(B \lambda) K_{0}(B \lambda) K_{1}(A \lambda) \lambda}{2 A} \\
- & \frac{B \kappa I_{0}(B \lambda) I_{1}(A \lambda) K_{0}(B \lambda) K_{1}(A \lambda) \lambda^{2}}{A}-\frac{B I_{1}(A \lambda) I_{1}(B \lambda) K_{1}(A \lambda) K_{1}(B \lambda) \lambda^{2}}{A} \\
- & \frac{B \kappa I_{1}(A \lambda) I_{1}(B \lambda) K_{1}(B \lambda) K_{1}(A \lambda) \lambda^{2}}{A}+\frac{A \lambda^{2}}{B}+\frac{\kappa^{2} I_{0}(B \lambda) I_{1}(B \lambda) K_{1}^{2}(A \lambda) \lambda}{2 A} \\
+ & \frac{\kappa I_{0}(B \lambda) I_{1}(B \lambda) K_{1}(A \lambda) \lambda^{2}}{A}+\frac{I_{0}(B \lambda) I_{1}(B \lambda) K_{1}^{2}(A \lambda) \lambda}{2 A} \\
+ & \frac{\kappa I_{1}(A \lambda) I_{1}(B \lambda) K_{0}(B \lambda) K_{1}(A \lambda) \lambda}{A}+\frac{I_{1}(A \lambda) I_{1}(B \lambda) K_{0}(B \lambda) K_{1}(A \lambda) \lambda}{2 A} \\
- & \frac{\kappa^{2} I_{1}^{2}(A \lambda) K_{0}(B \lambda) K_{1}(B \lambda) \lambda}{2 A}-\frac{\kappa I_{1}^{2}(A \lambda) K_{0}(B \lambda) K_{1}(B \lambda) \lambda}{A} \\
- & \frac{\kappa^{2} I_{0}(B \lambda) I_{1}(A \lambda) K_{1}(A \lambda) K_{1}(B \lambda) \lambda}{2 A}-\frac{\kappa I_{0}(B \lambda) I_{1}(A \lambda) K_{1}(A \lambda) K_{1}(B \lambda) \lambda}{A} \\
- & \frac{I_{0}(B \lambda) I_{1}(A \lambda) K_{1}(A \lambda) K_{1}(B \lambda) \lambda}{2 A}+\frac{\kappa^{2}}{4 A B}+\frac{\kappa}{A B}+\frac{3}{4 A B} \\
- & \frac{I_{1}^{2}(A \lambda) K_{0}(B \lambda) K_{1}(B \lambda) \lambda}{2 A}
\end{aligned}
$$

where

$$
H_{i j}(A \lambda, B \lambda)=K_{i}(A \lambda) I_{j}(B \lambda)+(-1)^{i+j+1} I_{i}(A \lambda) K_{j}(B \lambda) \quad(i, j=0,1) .
$$

$S_{1}-S_{4}$ appearing in Eq. (15) are given as:

$$
\begin{array}{ll}
S_{1} & =\frac{1}{(\kappa+1)} \int_{a}^{b} f(t) t g_{1} \mathrm{~d} t, \quad S_{2}=\frac{1}{(\kappa+1)} \int_{a}^{b} f(t) t b_{1} \mathrm{~d} t, \\
S_{3} & =\frac{1}{(\kappa+1)} \int_{a}^{b} f(t) t a_{1} \mathrm{~d} t, \quad S_{4}=\frac{1}{(\kappa+1)} \int_{a}^{b} f(t) t d_{1} \mathrm{~d} t,
\end{array}
$$

where

$$
\begin{aligned}
a_{1}= & -4\left(t I_{0}(A \lambda) K_{0}(t \lambda)-A I_{1}(A \lambda) K_{1}(t \lambda)\right) \lambda^{2} \\
& +\frac{4\left(t I_{1}(A \lambda) K_{0}(t \lambda)-A I_{0}(A \lambda) K_{1}(t \lambda)\right) \lambda}{A}+\frac{2(\kappa+1) I_{1}(A \lambda) K_{1}(t \lambda)}{A} \\
b_{1}= & -2 t \lambda I_{0}(t \lambda) K_{0}(B \lambda)-(\kappa+1) I_{1}(t \lambda) K_{0}(B \lambda)+2 B \lambda I_{1}(t \lambda) K_{1}(B \lambda) \\
d_{1}= & \frac{1}{2} \lambda^{2}\left(A I_{0}(A \lambda) K_{1}(t \lambda)-t I_{1}(A \lambda) K_{0}(t \lambda)\right) \\
g_{1}= & (\kappa+1) I_{1}(t \lambda) K_{1}(B \lambda)-2 \lambda\left(t I_{0}(t \lambda) K_{1}(B \lambda)-B I_{1}(t \lambda) K_{0}(B \lambda)\right)
\end{aligned}
$$

\section{References}

[1] Erdol R, Erdogan F. A thick-walled cylinder with an axisymmetric internal or edge crack. J Appl Mech 1978;45:281-6.

[2] Sneddon IN, Welch JT. A note on the distribution of stress in a cylinder containing penny-shaped crack. Int J Engng Sci 1963;1:411-9.

[3] Gupta GD. The analysis of the semi-infinite cylinder problem. Int J Solids Struct 1974;10:137-48.

[4] Delale F, Erdogan F. Stress intensity factors in hollow cylinder containing a radical crack. Int J Fract 1982;20:251-65.

[5] Nied HF, Erdogan F. The elasticity problem for a thick-walled cylinder containing a circumferential crack. Int J Fract 1983;22:277-301.

[6] Benthem JP, Minderhoud P. The problem of the solid cylinder compressed between rough rigid stamps. Int J Solids Struct 1972;8:1027-42.

[7] Artem HSA, Gecit MR. An elastic hollow cylinder under axial tension containing a crack and two rigid inclusions of ring shape. Comput Struct 2002;80:2277-87. 
[8] Birinci A. Axisymmetric crack problem of a thick-walled cylinder with cladding. Int J Engng Sci 2002;40:1729-50.

[9] Uyaner M. Dugdale-model for a ring-shaped crack in a transtropic thick walled cylinder. Z Angew Math Mech 2004;84:797-806.

[10] Aydin L. Investigation of stress intensity factors in an elastic cylinder under axial tension with a crack of ring-shape. Msc thesis, İzmir Institute of Technology, İzmir, Turkey; 2005.

[11] Watson GN. A treatise on the theory of bessel functions. London: Cambridge University Press; 1995.

[12] Muskhelishvili NI. Singular integral equations. Holland: Noordhoff; 1953.

[13] Artem HSA. Problem of cracked infinite hollow cylinder with two rigid inclusions. PhD thesis, Middle East Technical University, Ankara, Turkey; 1999.

[14] Stroud AH, Secrest D. Gaussian quadrature formulas. New York: Prentice-Hall; 1966. 